\title{
Fast Virtual Stenting with Deformable Meshes: Application to Intracranial Aneurysms
}

\author{
Ignacio Larrabide ${ }^{1,2}$, Alessandro Radaelli ${ }^{1,2}$, and Alejandro Frangi ${ }^{1,2}$ \\ Center for Computational Imaging and Simulation Technologies in Biomedicine \\ (CISTIB) \\ ${ }^{1}$ Networking Research Center on Bioengineering, Biomaterials and Nanomedicine \\ (CIBER-BBN), 08003 Barcelona, Spain \\ ${ }^{2}$ Universitat Pompeu Fabra, Circumval.lació 8, 08003 Barcelona, Spain
}

\begin{abstract}
Intracranial stents are medical devices that are becoming increasingly popular in the treatment of intracranial aneurysms. A methodology that predicts the released stent configuration prior to intervention has the potential to support the physician in the selection of the optimal approach for a specific patient. This paper proposes a fast virtual stenting technique based on constrained simplex deformable models that is able to virtually release stents in arbitrarily shaped vessel and aneurysm models. The technique effectively embeds the geometrical properties of the stent (cell design, strut size and shape and angles between struts) and achieves favorable execution times of the order of one minute.
\end{abstract}

Keywords: Virtual stenting, intracranial aneurysm, deformable meshes.

\section{Introduction}

Intracranial aneurysms are pathological dilatations of cerebral vessels, whose rupture leads to catastrophic complications such as diffuse bleeding in the brain cavities. Intracranial aneurysms may be treated using either surgical clipping or endovascular treatment, the latter primarily performed by coil embolization. Particular interest has been recently given to the use of intracranial stents as flow diverters. It has been suggested that the use of a stent alone may be enough to provide hemodynamic resistance to blood inflow and outflow into the aneurysm sac, thus promoting thrombosis and consequent normalization of the blood flow in the parent vessel [1]. In particular, the interplay between the geometry of the parent vessel and the stent design is key to achieve an acceptable conformability of the stent to the vessel wall [2]. A methodology for estimating the released configuration of a stent in a patient-specific geometry would be welcomed by interventional radiologists for treatment planning and training. Such virtual stent release method should be able to consider the geometrical properties of the stent (i.e., cell design, strut size and shape, angles between struts) and realistically predict the stent shape after its release in an arbitrarily shaped cerebral vessel or aneurysm with low computational cost. This is an important component that would facilitate the solution of a number of biomechanical problems, 
including stent stress analysis, stent-vessel structural interaction and haemodynamic alteration due to stent release. A first approach to virtual stent release based on deformable models was recently proposed by Appanaboyina et al. 3 . Although this technique considers the specific design of a stent, physical characteristics such as length of and angle between struts are not preserved and a realistic stent configuration may not be guaranteed especially in regions of high curvature. Similar limitations were found for the method proposed by FlórezValencia et al. 4], who suggest the use of simplex deformable meshes. Starting from an initial stent mesh placed along the centerline of a vessel, this approach achieves a smooth and regular mesh representation throughout the deformation process. Nevertheless, neither stent geometrical properties nor stent design are considered. This paper introduces a virtual stent release method for real vessel geometries, where an extension of deformable models based on simplex meshes is proposed to represent geometrical information that constrains the stent deformation process.

\section{Methodology}

The main idea behind the proposed methodology is the use of a second order differential equation for moving the stent mesh under the effect of internal and external forces. Following the work of Montagnat and Delingette [5, we use deformable simplex meshes with constraints. In particular, their deformation can be expressed using a finite difference discretization of the form

$$
\mathbf{p}_{i}^{\tau+1}=\mathbf{p}_{i}^{\tau}+(1-\widetilde{\gamma})\left(\mathbf{p}_{i}^{\tau}-\mathbf{p}_{i}^{\tau-1}\right)+\widetilde{\alpha} \mathbf{f}_{i n t}\left(\mathbf{p}_{i}^{\tau}\right)+\widetilde{\beta} \mathbf{f}_{e x t}\left(\mathbf{p}_{i}^{\tau}\right) .
$$

where $\mathbf{p}_{i}$ is a point of the simplex mesh, $\tau$ is the iteration number, $\mathbf{f}_{\text {int }}$ and $\mathbf{f}_{e x t}$ are the internal and external forces acting on the stent and $\widetilde{\gamma}, \widetilde{\alpha}$ and $\widetilde{\beta}$ are weighting parameters. In particular, we will use 2 -simplex meshes, meaning that each node of the mesh is connected to exactly 3 neighbors. In addition, we use a free boundary condition and details on the initial condition is provided in in a later section. The reader is referred to [5] for complementary definitions and additional information on simplex meshes.

Stent Mesh Representation. Standard simplex meshes are not appropriate for representing stents as stent geometries usually do not comply with the restrictions of a 2-simplex mesh (i.e., 3 neighbors per vertex). To overcome this limitation, we extend the main simplex mesh with information on the stent design. A simplex mesh is defined by a set of points and their connectivity. The implicit information carried over by the simplex mesh (i.e., surface curvature, surface normal and barycentric coordinates of the nodes) is used to guide its deformation while preserving regularity. Using the same points of the simplex mesh, a second background mesh is defined over the domain spanned by the simplex mesh. The background mesh contains geometrical information specific to the stent being released, including strut layout, strut length, angle between the struts, etc. 

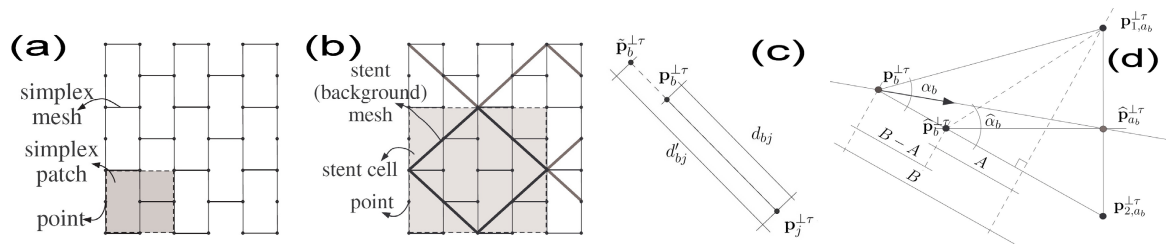

Fig. 1. Detail of simplex (a) and background (b) mesh. Figure (c) depicts how the strut length force $\left(\mathbf{f}_{\text {length }}\right)$ is the computed and figure $(\mathrm{d})$ the same for the angle force $\left(\mathbf{f}_{\text {angle }}\right)$. In figure (d), the notation $A=\left\|\widehat{\mathbf{p}}_{b}^{\perp \tau}-\mathbf{p}_{1, a_{b}}^{\perp \tau}\right\| \cos \left(\widehat{\alpha}_{b}\right)$ and $B=\left\|\mathbf{p}_{b}^{\perp \tau}-\mathbf{p}_{1, a_{b}}^{\perp \tau}\right\| \cos \left(\alpha_{b}\right)$ was considered for clarity.

Background Mesh. The background mesh is composed of a subset of points in the simplex mesh that carry information such as neighboring stent points that form the struts, stent forces applying to the point, stent geometrical description at the point, etc., that are used to define the restrictions on the deformation of the simplex mesh points. Geometrical characteristics of the stent released outside a vessel (default or "free" stent shape) may be easily obtained from a $\mu$-CT scan of the stent or, preferably, directly from the Computer Aided Design (CAD) models available to the stent manufacturer. In particular, we consider the stent cell design, the strut length and the angles between the struts as geometrical constraints. As most stents have a repeating cell design, the stent is modeled as a set of cells which, in turn, are composed of simplex mesh patches (Figure 1). This approach allows to map any stent design and characteristics on the whole simplex mesh using a simple repetition process.

Stent Geometrical Constraints. We consider three geometrical constraints: the stent design, the stent struts length, and the characteristic angles between struts in the default or "free" stent shape. The last two constraints are used to apply forces on the stent mesh points that lead to a deformation compliant with the geometrical properties of the stent. The introduction of such constraints is motivated by the following two aspects. First, these parameters are enough to describe, macroscopically, the stent geometry, provided that we are not interested in structural analysis of the stent (where additional information like strut cross sectional shape and stent endings on the proximal and distal side would be required). Second, this information is relatively easy to obtain. It is also important to note that this method does not force that the restrictions are fully satisfied, as the stent-specific properties are just used as soft constraints. The reason for using this simplification is that the stent shape constraints are measured for the free stent shape released outside the vessel. When released inside a vessel, the stent will not recover the same exact configuration. For this reason, the use of soft constraints, where a balance between internal and external forces is promoted, seems more appropriate.

Stent Deformation Model. We introduce a new stent shape-constraining force that encodes the geometric constraints introduced by a specific stent design. 
The background mesh is created by defining its connectivity over a subset of points of the simplex mesh $\mathbf{p}_{i}^{b} \in \mathcal{P}^{b} \subset \mathcal{P}$. Then, we can define $\mathcal{S}$ as the set of struts that form the stent. With this in mind, we propose to add a shapeconstraining force term to Eq. 1 that accounts for the shape constraints of the struts as introduced in Section 2 Adding this new term, Eq. 1 reads

$$
\begin{aligned}
\mathbf{p}_{i}^{\tau+1}= & \mathbf{p}_{i}^{\tau}+(1-\widetilde{\gamma})\left(\mathbf{p}_{i}^{\tau}-\mathbf{p}_{i}^{\tau-1}\right)+ \\
& \widetilde{\alpha}\left(\mathbf{f}_{\text {smooth }}\left(\mathbf{p}_{i}^{\tau}\right)+\widetilde{\chi} \mathbf{f}_{\text {length }}\left(\mathbf{p}_{b}^{\tau}\right)+\widetilde{\varepsilon} \mathbf{f}_{\text {angle }}\left(\mathbf{p}_{b}^{\tau}\right)\right)+\widetilde{\beta} \mathbf{f}_{\text {ext }}\left(\mathbf{p}_{i}^{\tau}\right) .
\end{aligned}
$$

External forces: An attracting force acting on the stent mesh towards the vessel wall is considered. This force acts in the mesh normal direction and is proportional to the distance to the vessel wall. Then, $\mathbf{f}_{e x t}\left(\mathbf{p}_{i}\right)=b_{s i} d\left(\mathbf{n}_{i}, \mathbf{p}_{i}\right) \mathbf{n}_{i}$, where $d\left(\mathbf{n}_{i}, \mathbf{p}_{i}\right)$ is the distance between $\mathbf{p}_{i}$ and the vessel wall in the direction of the mesh normal. One of the most relevant applications of the stent release method is in arteries with aneurysms. In the case of real stents, the stent stiffness avoids the stent from entering the aneurysm sac. To simulate this behavior we use of a variable $b_{s i}$. Let us illustrate this idea. The stent mesh is considered as a set of rings, and the center of mass $\mathbf{C}_{s}$ of each one is computed. Using $\mathbf{C}_{s}$, the average radius of section $\bar{r}_{s}=\sum_{k=1}^{N P_{s}}\left\|\mathbf{p}_{k}-\mathbf{C}_{s}\right\| / N P_{s}$ is calculated, where $N P_{s}$ is the number of points in each ring. Then, $b_{s i}$ will be zero if $\left\|\mathbf{p}_{k}-\mathbf{C}_{s}\right\|>\delta * \bar{r}_{s}$ and one otherwise. The value $\delta$ depends on the time step size selected, where for smaller time steps, a smaller $\delta$ value should be selected, ensuring a uniform deformation of the points within the same ring. We have found experimentally that the best results were obtained for values of $\delta$ between 0.05 and 0.20 (i.e., between 5 and $20 \%$ ). The effect of this parameter is to maintain all the points laying on the same ring at similar distance from the corresponding center of mass. To balance the influence of internal and external forces, a value of $\widetilde{\beta}=1-\widetilde{\alpha}$ with $0<\widetilde{\alpha} \leq 1$ was considered, where the best results where obtained for $\widetilde{\alpha}=0.5$. The external forces are stopped when a given mesh point is closer than a given value $\epsilon$ to the vessel wall.

Smoothing forces: For the smoothing internal forces, the same terms and parameters originally proposed by Montagnat and Delingette [5] are used.

Strut length force: We define the strut length constraint force at point $\mathbf{p}_{b}^{\tau}$ as $\mathbf{f}_{\text {length }}\left(\mathbf{p}_{b}^{\tau}\right)$, namely $\mathbf{f}_{\text {length }}\left(\mathbf{p}_{b}^{\tau}\right)=\left(\tilde{\mathbf{p}}_{b}^{\perp \tau}-\mathbf{p}_{b}^{\perp \tau}\right)$ with, $\tilde{\mathbf{p}}_{b}^{\perp \tau}=\mathbf{p}_{b}^{\perp \tau}+\left(\sum_{j \in \mathcal{N}_{b}(i)} \zeta_{b j}\right.$ $\left.\left(\mathbf{p}_{b}^{\perp \tau}-\mathbf{p}_{j}^{\perp \tau}\right) /\left\|\mathbf{p}_{b}^{\perp \tau}-\mathbf{p}_{j}^{\perp \tau}\right\|\right) /\left|\mathcal{N}_{b}(i)\right|$, being $\mathcal{N}_{b}(i)$ the set of neighbors of point $\mathbf{p}_{b}^{\tau}$ in the background mesh, $|\cdot|$ is the cardinality of a set, and where $\zeta_{b j}=d_{b j}^{\prime}-d_{b j}$. In the former, $d_{b j}^{\prime}$ is the reference length of the strut between points $\mathbf{p}_{b}^{\tau}$ and $\mathbf{p}_{j}^{\tau}$, and $d_{b j}$ the actual length at time $\tau$. The objective of the length force is to preserve the stent length (observed in the released stent) at the end of the deformation process (Figure 1- c).

Strut angle force: We define the strut angle constraint force at point $\mathbf{p}_{b}^{\tau}$ as $\mathbf{f}_{\text {angle }}\left(\mathbf{p}_{b}^{\tau}\right)$, namely $\mathbf{f}_{a n g l e}\left(\mathbf{p}_{b}^{\tau}\right)=\left(\widehat{\mathbf{p}}_{b}^{\perp \tau}-\mathbf{p}_{b}^{\perp \tau}\right)$, with $\widehat{\mathbf{p}}_{b}^{\perp \tau}=\mathbf{p}_{b}^{\perp \tau}+\left(\sum_{a_{b} \in \Lambda(b)} \phi_{a_{b}}\right.$ 


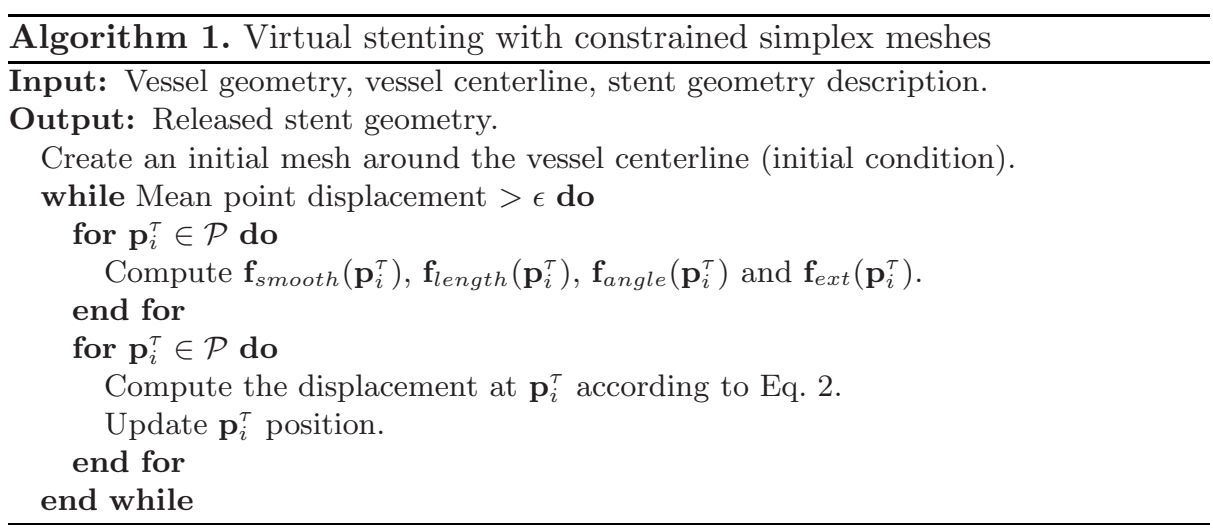

$\left.\left(\mathbf{p}_{b}^{\perp \tau}-\widehat{\mathbf{p}}_{a_{b}}^{\perp \tau}\right) /\left\|\mathbf{p}_{b}^{\perp \tau}-\widehat{\mathbf{p}}_{a_{b}}^{\perp \tau}\right\|\right) /|\Lambda(b)|$ being $\Lambda(b)$ the set of angles $a_{b}$ formed between $\mathbf{p}_{b}^{\tau}$ and two neighbors, $\widehat{\mathbf{p}}_{a_{b}}^{\perp \tau}=\mathbf{p}_{1, a_{b}}^{\perp \tau}+\mathbf{p}_{2, a_{b}}^{\perp \tau} / 2$, and $\phi_{a_{b}}=|B|-|A|$, where $\alpha_{b}=\mathbf{p}_{1, a_{b}}^{\perp \tau} \widehat{\mathbf{p}_{b}^{\perp \tau}} \mathbf{p}_{2, a_{b}}^{\perp \tau}$ is the value (in radians) of the angle $a_{b}$ and $\widehat{\alpha}_{b}$ is the corresponding reference value. This force is intended to preserve the angle between the struts (observed in the released stent) at the end of the deformation process (Figure 1-d). In order to balance the influence of both shape forces $\mathbf{f}_{\text {length }}\left(\mathbf{p}_{b}^{\tau}\right)$ and $\mathbf{f}_{\text {angle }}\left(\mathbf{p}_{b}^{\tau}\right)$, we consider $1=\tilde{\chi}+\widetilde{\varepsilon}$. The best results where obtained for $\tilde{\chi}$ between 0.6 and 0.9. In order to release a stent in a target vessel, we first need to place the stent inside an appropriate vascular segment or, in other words, generate an initial condition. To this goal, we use the centerline of the target vessel. The initial mesh is then created in three steps: first the simplex mesh points, second the simplex mesh connectivity using those points, and finally the stent (background) mesh. To represent an arbitrary stent design, we use the information of a cell (see Fig. 1) which is repeated over the stent. Regarding the mesh displacement, the simplex and stent mesh forces are computed sequentially, and the position of each mesh point is updated based on these forces. The proposed approach is described in Algorithm 1.

\section{Results}

In order to evaluate the methodology, a first series of tests is performed using a simple synthetic vessel geometry. The stent geometry used (presented in Figure 2) is composed of rhomboidal stent cells formed of 4 struts (repeated 10 times around the stent and 9 times over the stent length). The strut length constraint considered is $d_{b j}^{\prime}=0.4 \mathrm{~mm}$ (equal for the 4 struts in the cell) and the angle constraints considered are $\widehat{\alpha}_{1}=\widehat{\alpha}_{2}=\widehat{\alpha}_{3}=\widehat{\alpha}_{4}=\pi / 2$. In order to verify the correctness of the deformation, we introduce two indices that provide an assessment of the compliancy of the stent mesh to the constraints under consideration. We define the strut length mean error $\bar{e}_{\text {length }}=\left(\sum_{j \in \mathcal{N}_{b}(i)}\left\|d_{b j}-d_{b j}^{\prime}\right\| / d_{b j}^{\prime}\right) /\left|\mathcal{N}_{b}(i)\right|$, and the strut angle mean error $\bar{e}_{\text {angle }}=\left(\sum_{a_{b} \in \Lambda(b)}\left(\left|\cos \left(\alpha_{b}\right)\right|-\left|\cos \left(\widehat{\alpha}_{b}\right)\right|\right)\right) /|\Lambda(b)|$. 

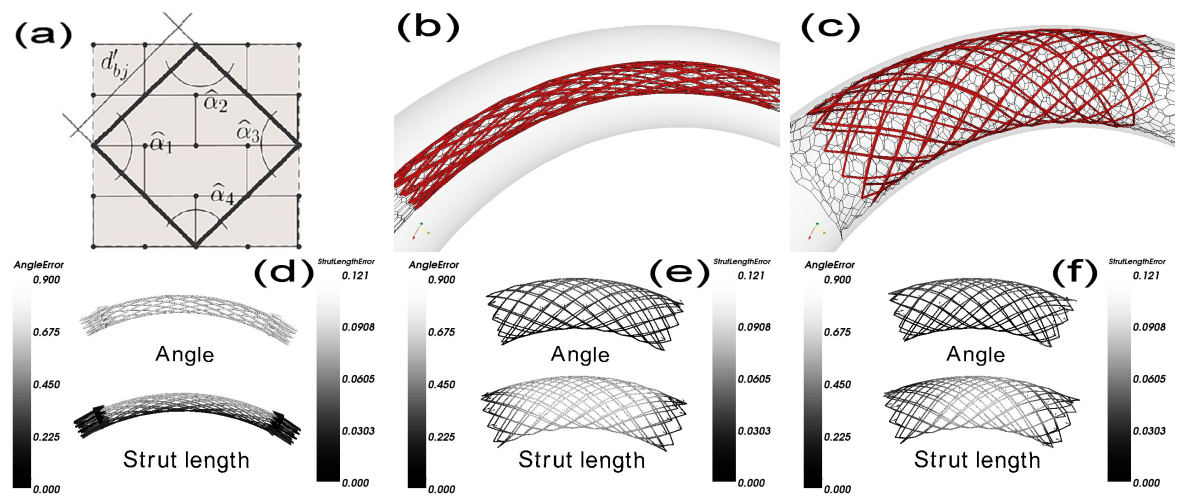

Fig. 2. Stent design and stent mesh constraints for a simple stent case (a). In this case, $\widehat{\alpha}_{1}=\widehat{\alpha}_{2}=\widehat{\alpha}_{3}=\widehat{\alpha}_{4}=\pi / 2$ and $d_{b j}^{\prime}=0.4 \mathrm{~mm}$ was considered. Synthetic vessel (straight tube, with radius 1 ) with the stent in its initial (b) and final (c) position (radius 0.2) together with the simplex mesh are presented on the left. Second row: Stent mesh snapshots at 1,75 , and 150 iterations. Color codes the values of $\bar{e}_{\text {angle }}$ and $\bar{e}_{\text {length }}$.
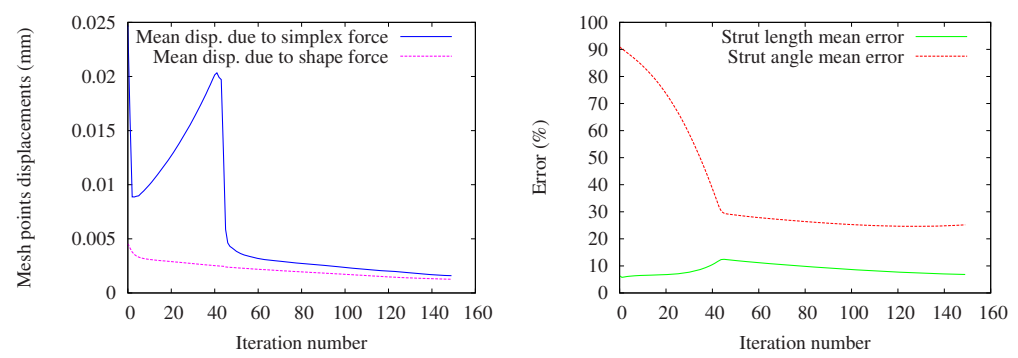

Fig. 3. The plot on the right presents the evolution of mean mesh point displacement due to simplex (external and smooth) and shape forces. On the left, the plot represents $\bar{e}_{\text {length }}$ and $\bar{e}_{\text {angle }}$.

The release was performed using $\widetilde{\alpha}=0.6$ and $\tilde{\chi}=0.85$. The external force (i.e., vessel attracting force) was considered null, i.e. $\mathbf{f}_{e x t}\left(\mathbf{p}_{i}\right)=0$ when points where closer than $0.05 \mathrm{~mm}$ from the surface $(\epsilon=0.05 \mathrm{~mm}), \delta=0.2$ and an initial stent radius of $0.2 \mathrm{~mm}$ was used. All the tests mentioned in this work were executed on an Intel (B) Core ${ }^{\mathrm{TM}}$ Duo CPU T7300 2.00GHz with $2 \mathrm{~Gb}$ of memory and no parallelization (i.e., multi-threading) was used. A run of 150 iterations required 105 seconds. Figure 2 shows selected snapshots for the deformation sequence (1, 75 and 150 iterations, subfigure d, e and f respectively). In each of these images, the shape on top represents the stent colored by $\bar{e}_{\text {angle }}$ with a vector glyph representing $\mathbf{f}_{\text {angle }}$ and the shape on the bottom shows the same for $\bar{e}_{\text {length }}$ and $\mathbf{f}_{\text {length }}$. It can be observed that the angle and length error both decrease as the algorithm evolves. After the stent mesh reaches the vessel wall, the shape constraints continue to act over the mesh improving its compliance. We 

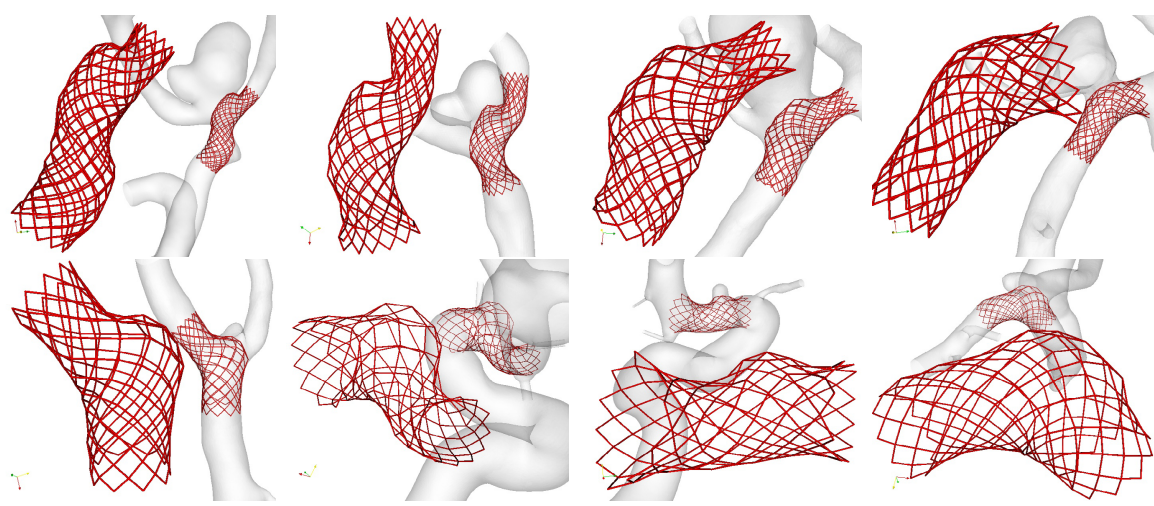

Fig. 4. The images present the result for the release of the previously described stent in different (real) vessel geometries with aneurysms. In the background is presented the geometry under consideration with the released stent inside it.

can observe that $\bar{e}_{\text {angle }}$ is smaller over the part of the mesh laying in the inside of the vessel (where the stent cells are more open) and is greater in the outside (where cells remain more closed). For the $\bar{e}_{\text {length }}$, we observe the larger error on the inside of the vessel curve, where compression would be expected. The plots on Figure 3 show the mean displacement of each mesh point (for the simplex and stent meshes), and $\bar{e}_{\text {length }}$ and $\bar{e}_{\text {angle }}$ as function of iteration number. It can be observed that until iteration 50 (when the vessel wall is reached), the simplex mesh points move considerably. After that, mainly the internal forces are acting over the mesh and they stabilize until almost no movement is observed in the mesh. After few iterations, both forces balance and the error stabilizes.

Stenting of real geometries. In a further set of experiments, the stent release method was tested in patient-specific vessel geometries extracted from medical images. All the geometry models correspond to intracranial aneurysms segmented and reconstructed from 3DRA (3D rotational angiography) images. Figure 4 shows the geometry models and the corresponding released stent geometry, using the simple design previously described. The average time required for the release in these geometries was 66.88 seconds with a standard deviation of 25.37 seconds. The average number of iterations was 72 with a standard deviation of 10. Overall, this methodology requires a low computational cost and may be considered quasi real time, achieving execution times of approximately 1 minute for real vessel geometries on a standard computer configuration.

\section{Discussion and Future Work}

We presented a methodology for fast virtual stenting based on deformable simplex meshes and showed its application to intracranial aneurysms. The motivation behind our proposed methodology was threefold: versatility to incorporate 
the geometrical properties of real stents, the ability to virtually release the stent in real vessel geometries, and the need for a low computational cost yielding fast execution times.

It is important to mention that drastically changing the stent cell design and size (e.g., for a $2 \times$ size vessel) may require the tuning of the algorithm parameters $(\widetilde{\beta}, \widetilde{\chi}$ and $\delta)$. This aspect deserves more careful and detailed consideration and is currently subject of further analysis.

Another important issue is performing a more exhaustive validation. In particular, we are currently working along two main research lines: a) qualitative comparison of $\mu$-CT images of commercial stents released in anatomically realistic silicon phantoms with virtually released stents in the reconstructed models of the same phantoms, b) qualitative comparison of digital substraction angiographic images showing contrast diffusion in silicon phantoms geometries before and after stenting with virtual angiography using Computational Fluid Dynamics in the reconstructed phantom models before and after virtual stenting, and c) same as b) but with real patient data.

Acknowledgements. The authors would like to thank E. Vivas, MD, from the NAT-HGC (Barcelona, Spain) for providing the 3DRA data and clinical background. This work was partially supported within the CENIT-CDTEAM project funded by the Spanish CDTI and partly within the framework of the @neurIST Project (IST-2005-027703), which is co-financed by the European Commission within the IST Program of the Sixth Framework Programm.

\section{References}

1. Jamous, M.A., Satoh, K., Matsubara, S., Satomi, J., Nakajima, N., Uno, M., Nagahiro, S.: Ischemic basilar artery dissecting aneurysm treated by stenting only Case report. Neurol. Med. Chir. 44(2), 77-81 (2004)

2. Ebrahimi, N., Claus, B., Lee, C.Y., Biondi, A., Benndorf, G.: Stent conformity in curved vascular models with simulated aneurysm necks using flat-panel CT: An in vitro study. Am. J. Neuroradiol 28(5), 823-829 (2007)

3. Appanaboyina, S., Mut, F., Lohner, R., Putman, C.M., Cebral, J.R.: Computational fluid dynamics of stented intracranial aneurysms using adaptive embedded unstructured grids. Int. J. Numer. Meth. Fluid. 57(5), 475-493 (2007)

4. Valencia, L.F., Montagnat, J., Orkisz, M.: 3D Graphical models for vascular-stent pose simulation. Innovations Technol. Biol. Med. 28(2), 65-71 (2007)

5. Montagnat, J., Delingette, H.: 4D deformable models with temporal constraints: application to $4 \mathrm{D}$ cardiac image segmentation. Med. Image Anal. 9, 87-100 (2005) 